\title{
Effects of Tonifying Kidney and Spleen, Detoxification of Throat for Th1 /Th2 of IgA Nephropathy Model Rats
}

\author{
Di ZOU ${ }^{1, a}$, Dong-mei ZHANG ${ }^{2}$,Yin-ping $\mathrm{WANG}^{3}$ and Shou-lin \\ ZHANG ${ }^{4 b^{2}}$
}
${ }^{1,2,3,4}$ Department of Nephrology, the First Affiliated Hospital to Changchun University of Chinese Medicine, Changchun, Jilin, China,130021.

aaggiezou@163.com , bshoulin-z@163.com

* Shou-lin ZHANG

Keywords: Tonifying kidney and spleen, Detoxification of throat, $\lg A$ nephropathy, Cytokines, Th1 / Th2

\begin{abstract}
Purposes:to investigate the effects of tonifying kidney and spleen, detoxification of throat for Th1 /Th2 of IgA nephropathy model rats.Methods : 72 rats were randomly divided into blank group, model group, Tripterygium wilfordii multi glycoside tablet group (control group), tonifying kidney and spleen, detoxification of throat the medicine low, middle and high dose group, referring to reports of Huiyu Lu,such as the method of preparation of animal model, the fluorescent light semi quantitative method for detection of rat renal tissue membrane $\operatorname{IgA}$ deposition, enzyme linked immunosorbent assays (ELISA) for detection of ratexpression level of serum cytokines interleukin prime (IL) IL-2, IL-4, IL-10, interference prime (INF) $-\gamma$. Results:After the model results showed varying degrees of poor appetite, hair messy, weight loss, kidney mesangial IgA deposition. The treatment group rat IgA deposition has been reduced. Compared with the blank group, the model group rats serum IL-2 and INF- $\gamma$ levels were significantly lower $(P<0.05)$ and IL-4, IL-10 levels were significantly increased $(P<0.05)$ and Th1/Th2 ratio appears to drift. Compared with model group, the serum levels of the treatment group IL-2 and INF- $\gamma$ levels significantly increased $(P<0.05)$ and IL-4, IL-10 levels decreased significantly $(P<0.05)$ and Th1/Th2 drift improved. The traditional Chinese medicine group and the control group had no significant difference $(P>0.05)$. Conclusion:IgAN model rat cytokine IL-2, IL-4, IL-10, INF- yexpression disorder and Th1/Th2 drift and the method of tonifying kidney and spleen, detoxification of throat can adjust the expression levels of cytokines IL-2, IL-4, IL-10 and INF- $\gamma$, and correct theTh2 / Th1 ratio drift.
\end{abstract}

\section{Introduction}

Incidence of IgA nephropathy (IgAN) in China was $45 \%$ to $50 \%$ [1], about $15 \%$ $40 \%$ of IgAN patients of which will be the end stage of renal disease in 10 years[2,3]. Professor Jixue Ren thought the pathogenesis core of disease is the spleen and kidney deficiency and pharyngeal infection is the source of the incidence.It was proposed to invigorate the kidney and spleen and detoxification of throat treatment of the disease which was clinical used for 40 years. The curative effect is remarkable. This paper aims to further explore the mechanism of the therapy. 


\section{Materials and Methods}

\section{Experime ntal Animals}

72 SD male rats which body weight was $(180 \pm 20)$, the urine red cell count and urinary protein qualitative test were negative in all rats.

\section{Experime ntal Medicine}

Tripterygium glycosides tablet: Shanghai Fudan Fuhua Co.,Ltd production, atch number: Chinese medicine quasi word Z31020415. Tonifying kidney and spleen, detoxification of throat : Radix Rehmanniae $20 \mathrm{~g}$, as tragalus root $20 \mathrm{~g}$,Cornus $20 \mathrm{~g}$, yam 20g, Mao Gen 50g, soil Fuling 30g, Pu Huang $15 \mathrm{~g}$ (including fried), puffball $15 \mathrm{~g}$, wild buckwheat $30 \mathrm{~g}$,Kadsura Root-bark $15 \mathrm{~g}$, oroxylum Butterfly $15 \mathrm{~g}$, licorice $5 \mathrm{~g}$. Unified system of pharmacy in the Affiliated Hospital of Changchun University of Traditional Chinese Medicine.

\section{Reagents and Instruments}

Fluorescence microscope TXM-500C, Shanghai TianSheng Instrument Co., Ltd.Full automatic microplate reader 318C. Shanghai ZhuoFei laboratory equipment Co., ltd. WhiteInterleuk in prime (IL) - 2, IL-4, IL-10 and IFN gamma (INF gamma), enzyme linked immunosorbent (ELISA) detection reagent box, provided by Shanghai Xinyu Biological Technology Co., Ltd. Bovine serum albumin (BSA) was provided by sigma company in America. FITC labeled Rabbit anti mouse IgA. Beijing Boosen Biological Technology Co., ltd.

\section{Animal Models Preparation}

Rats were fed one week after the purchase to adapt to the environment. Huiyu Lu [4]reported methods for the preparation of animal models.In ninth weeks each of the selected 2 renal tissue was taken from the kidney by fluorescence semi quantitative(fluorescence semi quantitative standards with reference to the domestic and foreign general five tier classification method[5]) of rat renal mesangial IgA deposition strength judgment. Model verification is successful, the general activity of rats were observed closely in the whole process.

\section{Animal Grouping and Administration}

The rats were divided into 6 groups by random.12 rats in each group, such as model group, blank group, Tripterygium wilfordii multi glycoside tablet group (control group), tonifying kidney and spleen,detoxification of throat low, middle, high dose group respectively.

Blank group: the model was made successfully.Giving $4 \mathrm{ml} / \mathrm{kg} / \mathrm{d}$ saline by gavage, once daily, continuing to the end of the 12 th week.Model group: since the 9 th week, giving physiological saline $4 \mathrm{ml} / \mathrm{kg} / \mathrm{d}$ orally, once daily, continuing to the end of the 12th week.Control group:after the model made successfully,giving Tripterygium wilfordii multi glycoside tablet $6 \mathrm{ml} / \mathrm{kg} / \mathrm{d}$, by gavage once daily continuing to the end of the 12th week.Low dose of Chinese medicine group: after the model made successfully, giving the decoction of Chinese Medicine $4 \mathrm{~kg} \mathrm{ml} / \mathrm{kg} / \mathrm{d}$ by gavage, containing $8 \mathrm{~g} / \mathrm{kg}$ of the TCM, once daily of the stomach and continuing to the end of the 12th week.Middle dose of Chinese medicine group: after the model made successfully, giving the decoction of Chinese Medicine $4 \mathrm{~kg} \mathrm{ml} / \mathrm{kg} / \mathrm{d}$ by gavage, containing $16 \mathrm{~g} / \mathrm{kg}$ of the TCM, once daily and continuing to the end of the 12 th week.High dose of Chinese medicine group: after the model made successfully, 
giving the decoction of Chinese Medicine $4 \mathrm{~kg} \mathrm{ml} / \mathrm{kg} / \mathrm{d}$ by gavage, containing $32 \mathrm{~g} / \mathrm{kg}$ of the TCM, once daily and continuing to the end of the 12 th week.

\section{Observation Index}

Observing the general state of the rats, modeling after 9 weeks in each group from two rats, using fluorescence semi quantitative detection of rat kidney IgA deposition, and expression levels by ELIS A method for detection of rat serum cell factor IL-2, IL-4, IL-10, INF- $\gamma$.

Statistical Methods Using SPSS13.0 software for single factor analysis of variance.

\section{Results}

\section{The General Condition}

The rats were killed nine. After modeling, rats appearing different degree of poor appetite, hair color messy,diarrhea,weight loss. When treatment was given, the appetite of the rats and the coat color has improved, weight gaining, which body weight growth faster in Chinese medicine group.

\section{IgA Deposition Degree}

After treatment groups rats immune fluorescence deposition rats in the blank group glomerular basement membrane zone and capillary walls were no $\operatorname{IgA}$ deposition (-), the model group rats have a strong diffuse mass like IgA deposition (++++), in Chinese medicine group and low, medium and high dose groups of $\operatorname{IgA}$ deposition degree different, but compared with model group, the intensity is significantly reduced, as shown in Figure 1.

Fig. 1 immune fluorescence deposition of rats in each group

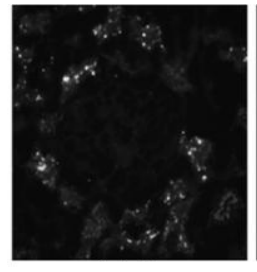

Blank group

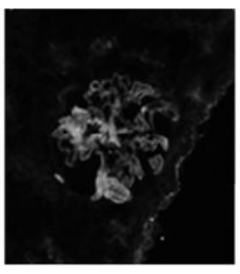

Model group

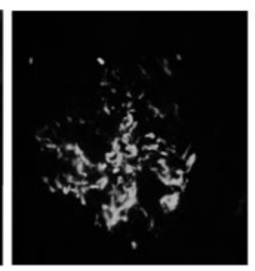

control group

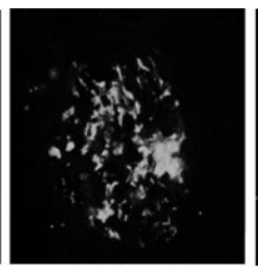

Low dose

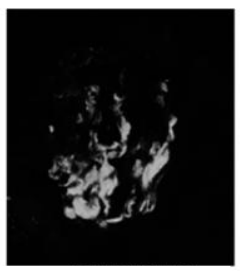

middle dose

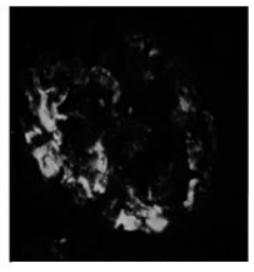

high dose

\section{The Expression of IL-2, IL-4, IL-10 and INF- $\gamma$}

In serum of the model group IL- 2 and INF- $\gamma$ were decreased compared with the blank group $(P<0.05)$, and the level of IL-10 and IL-4 was higher $(P<0.05)$ than that in the blank group.After tonifying kidney and spleen, detoxification throat method of Chinese medicine treatment, compared with the model group, level of the Chinese groups of IL-2 and INF- $\gamma$ was up-regulated, the level of IL-4,IL-10 being down regulated.Correcting the Th1/Th2 imbalance, which traditional Chinese medicine group improve degree and the control group showed no significant difference. as shown in table 1. 
Table 1 Comparison of expression levels of IL-2, IL-4, INF- $\gamma$ and IL-10 in serum of rats in each

\begin{tabular}{cccccc}
\multicolumn{5}{c}{$\operatorname{group}(\bar{x} \pm \mathrm{s}, \mathrm{n}=12)$} \\
\hline group & $\mathrm{IL}-2(\mathrm{ng} / \mathrm{ml})$ & $\mathrm{IL}-4(\mathrm{pg} / \mathrm{ml})$ & $\mathrm{IL}-10(\mu \mathrm{g} / \mathrm{L})$ & $\mathrm{INF}-\gamma(\mathrm{pg} / \mathrm{ml})$ & $\mathrm{INF}-\gamma / \mathrm{IL}-4$ \\
\hline Blank group & $4.84 \pm 0.89$ & $105.11 \pm 10.98$ & $51.48 \pm 8.97$ & $789.51 \pm 20.94$ & $7.12 \pm 0.54$ \\
Model group & $\left.2.45 \pm 1.41^{2}\right)$ & $159.23 \pm 11.14^{2)}$ & $81.64 \pm 9.15^{2)}$ & $453.36 \pm 13.61^{2)}$ & $4.08 \pm 0.21^{2)}$ \\
Control group & $3.49 \pm 0.67^{1)}$ & $106.32 \pm 10.32^{1)}$ & $53.16 \pm 8.31^{1)}$ & $731.67 \pm 19.36^{1)}$ & $7.12 \pm 0.54^{1)}$ \\
Low dose & $3.41 \pm 1.06^{1)}$ & $107.56 \pm 10.78^{1)}$ & $56.42 \pm 9.11^{1)}$ & $792.72 \pm 18.46^{1)}$ & $8.16 \pm 0.33^{1)}$ \\
Middle dose & $4.46 \pm 3.27^{1)}$ & $106.37 \pm 9.68^{1)}$ & $54.72 \pm 9.63^{1)}$ & $823.41 \pm 15.75^{1)}$ & $8.14 \pm 0.24^{1)}$ \\
High dose & $3.84 \pm 1.10^{1)}$ & $106.30 \pm 9.98^{1)}$ & $58.47 \pm 9.48^{1)}$ & $814.26 \pm 15.73^{1)}$ & $7.92 \pm 0.51^{1)}$ \\
\hline
\end{tabular}

Compared with model group: 1) $P<0.05$; compared with the blank group: 2) $P<0.05$

\section{Discussion}

The important pathogenesis of IgAN is IgA or IgA based immune complex deposition. It has been generally accepted that $\mathrm{T}$ helper cells divided into Th1 cells and $\mathrm{Th} 2$ cell subsets, which Th1 cells secreted interferon and IL-2 cytokine mediated cellular immune responses, and Th2 cells secreted IL- 4 and IL-10 cells due to sub humoral immune response. Th1 and Th2 cytokines will respectively induced Th0 cells to the respective cell subsets differentiation and promote the cell activation, and under normal circumstances, the Th cell subsets between is mutual constraints, various cytokines to another cell subsets and activation are inhibited, the Th1 and Th2 type cytokines influence and constrain each other resulting in immune responses play a regulatory role[6,7]. Therefore, the Th1 and Th2 subsets should be balanced, and the occurrence of imbalance is closely related to the occurrence of immune nephritis. Nogaki[8,9] found that the combined application of IL-4 and Th2 in mice could induce B cells to induce abnormal glycosylation of IgA cells. Previous studies also show that IgAN patients in active phase of Th1/Th2 imbalance, that INF- $\gamma$ down, IL-4 and IL-10, after remission is Th1/Th2 imbalance in corrective, namely INF- $\gamma$ up-regulated, IL-4 and IL-10 down showed that there was a Th1/Th2 imbalance in IgAN[10]. Therefore, it is worth further exploring the possibility of using the method to correct the imbalance of $\mathrm{Th} 2 / \mathrm{Th} 1$ for the treatment of IgAN, while the transmission and injection of exogenous cytokines of Th1 and Th2 cytokines to correct the Th1/Th2 imbalance[10], there are great difficulties, and the traditional Chinese medicine on the immune system with an important role in regulation.

Professor Jixue Ren, master of traditional Chinese medicine believes that the disease pathogenesis core of losses for the deficiency of the spleen and kidney, stagnation of pathogen throat is causative factors of the disease. As "ling shu" pointed out that "the kidney meridian of foot Shaoyin meridian, The straight from the renal tubular liver and diaphragm, into the lungs, through the throat, with the tongue". $\mathrm{V}$ isible throat is kidney lung collaterals, for access to the evils of the portal and toxin from the nose and mouth, entrenched in the throat, forming milk moth, from blood invasion in the kidney, Fuxie formation, long and to poison, living in the palace of kidney, forming a vicious cycle of throat, lung, kidney. Therefore, the occurrence of nephritis and recurrence and exacerbation of the disease and is closely related to the throat.Therefore Jixue Ren raised method of tonifying kidney and spleen, detoxification of throat.In summary, tonifying the kidney and strengthening the spleen, detoxification throat method in the treatment of IgAN has better clinical efficacy, consideration and regulation of cytokines IL-2, IL-4, IL-10, INF- $\gamma$ expression level and correcting Th1/Th2 imbalance is closely related. 


\section{Acknowledge ment}

This research was financially supported by project as follows:

Jilin Province Science and Technology Department of Natural Science Fund Project (No. 2015101214JC).

Jilin Province Education Department "Twelfth Five Year Plan" science and technology research project (Ji UNESCO Zi No. 65 of 2014);

Jilin Province Administration of traditional Chinese medicine science and technology project (No. 2014-zd7)

\section{References}

[1]Berger J,Hingais N. Intercapillary deposits of $\operatorname{IgA}-\operatorname{IgG}[\mathrm{J}] . J$ Urol Nephrol (Pans),1968;74(9):694- 5.

[2] Radford MG Jr,Donadio JV Jr, Bergstralh EJ, et al.Predicting renal outcome in IgA nephropathy[J].J Am Soc Nephrol,1997;8(2):199-207.

[3] D' Amico G. Natural history of id iopathic IgA nephropathy and factors pre-

dictive of disease outeome[J].Semin Nephrol, 2004;24(3):179-96.

[4] Lu Huiyu, Zhang Qiaoling, Jiang Xiaoyun, et al. Establishment of IgAN rat model (in Chinese)J. Journal of Chinese medical science, 2011;11(6):1264- 7.

[5] Liu Yanfang. Immunohistochemical method(in Chinese). Beijing: People's Medical Publishing House, 1990:56- 62.

[6] Kang H,Ahn KS,Cho C,et al.Immunomodulatory effect of Astragali Radix extract on murine Th1/Th2 cell lineage development[J].Biol Pharm

Bull,2004;27(12):1946- 50.

[7] Chen L,Martinez O,Ovebergh L,et al.Early up- regulation of Th2 cytokines and late surge of Th1 cytokines in an aopic dermatitis model[J],Clin Exp Immunol,2004;138(3):375- 87.

[8] Nogaki F,Muso E,Kobayashi I,et al.Interleukin 12 induces crescentic

glomerular lessions in a high IgA strain of ddY mice, independently of chang in IgA deposition[J].Nephron Dial Transplant,2000;15 (8):1146- 54.

[9] Chintalacharuvu SR,Yamashita M,Bagheri N,et al.T cell cytokine polarity as a determinant of immunoglobulin $\mathrm{A}(\operatorname{IgA})$ glycosylation and the severity of experimengtal IgA nephropathy[J].C lin Exp Immunol,2008;153(3):456- 62.

[10] Yu Dajun, Nie Lifang, Xu Yonggang, et al. Yiqi Zishen Granule on IgA nephropathy helper $\mathrm{T}$ lymphocyte Effect of lymphocyte subsets in the J. Chinese combine traditional Chinese and Western Medicine 2006;26(9):836- 8. 